\title{
Estudio de la adopción y el abandono de modelos de gestión de la calidad en centros gerontológicos de la CAPV ${ }^{1}$
}

\author{
Erlantz Allur \\ Escuela Universitaria de Estudios Empresariales de Donostia-San Sebastián, \\ Universidad del País Vasco (UPV/EHU)
}

\section{Iñaki Heras}

Escuela Universitaria de Estudios Empresariales de Donostia-San Sebastián, Universidad del País Vasco (UPV/EHU)

<inaki.heras@ehu.es>

\section{Martí Casadesús}

Escuela Politécnica Superior, Universitat de Girona

\begin{abstract}
Artikulu honetan aztertzen da ISO 9001 kalitateestandarra pertsona adinduentzako egoitzazerbitzuen alorrean, eta baita ere aztertzen dira aipatutako estandar horren egiaztapena uztearen kausa eta ondorioak bere alde egindako sektore honetako erakundeen artean. Hori dela-eta, bertan jasotzen dira EAEko adinduentzako 262 egoitzetan buruturiko inkestaren ondorio nagusiak. Azterlan kuantitatibo hori osatu zen zuzendari, arreta zuzeneko langile eta zentro horietako erabiltzaileen familiekin buruturiko izaera kualitatiboko 20 elkarrizketa baino gehiagotan oinarritutako beste azterlan batekin. Emaitzek nabarmentzen dute maiztasun gutxikoa izan dela ISO 9001 egiaztatzea utzitako kasuen kopurua sektore honetan, eta kalitatearen nazioarteko kudeaketa-eredu hori hoberen barneratu omen dute oinarriko motibazio gisara egiaztapen hori lortzea helburu ez zuten egoitzek.
\end{abstract}

\section{HITZ-GAKOAK:}

Kalitatearen kudeaketa, ISO 9001, egiaztapena, barneratzea, pertsona adinduentzako egoitzak, Euskadi.
Este artículo analiza la adopción del estándar de calidad ISO 9001 en el ámbito de los servicios residenciales para personas mayores, y examina también cuáles han sido las causas y consecuencias del abandono de la certificación de dicho estándar en aquellas organizaciones del sector que habían optado por él. Para tal fin, se recogen las principales conclusiones de una encuesta realizada en 262 residencias para personas mayores de la CAPV, estudio cuantitativo que fue complementado con otro de carácter cualitativo, basado en más de 20 entrevistas personales con directivos, personal de atención directa y familiares de los usuarios de los centros. Los resultados evidencian que el abandono de la certificación ISO 9001 ha sido poco frecuente en el sector, y que las organizaciones que mejor han interiorizado este modelo internacional de gestión de la calidad han sido las que no perseguían obtener el certificado como principal motivación.

\section{Palabras clave:}

Gestión de la calidad, ISO 9001, certificación, interiorización, residencias para personas mayores, País Vasco.

\footnotetext{
${ }^{1}$ Este artículo es resultado del Grupo de Investigación del Sistema Universitario Vasco IT763-13 / GIC12-158, financiado por el Gobierno Vasco. Los autores desean mostrar su agradecimiento a todas las personas entrevistadas en el trabajo de campo analizado en estas páginas.
} 


\section{Introducción}

Con cierto retardo respecto a su adopción en las organizaciones de los EE.UU., la corriente, movimiento o moda de gestión (managerial fad) de la calidad llegó a la Unión Europea. En algunos Estados miembros, como España o Italia, el paradigma de la gestión de la calidad se introdujo con mucha fuerza en sectores en los que la gestión y dirección de organizaciones no se encontraba muy profesionalizada; fue el caso del sector educativo, el sanitario y el de los servicios sociales. De esta forma, hace ya más de una década que los sistemas y modelos de gestión de la calidad comenzaron a considerarse como herramientas útiles y necesarias en las organizaciones asistenciales del ámbito sociosanitario.

Fruto de este proceso de difusión general, en el Estado español algunos modelos o estándares que en un inicio surgieron claramente enfocados al sector industrial -como el estándar internacional ISO 9001- experimentaron una amplia difusión en el sector sociosanitario, y sucedió lo mismo con otros modelos de gestión más genéricos, como el modelo de autoevaluación EFQM. Es más, en el ámbito del cuidado residencial a las personas mayores se desarrollaron modelos de gestión específicos, como la norma UNE 158001 para el sector residencial, un estándar que no parece haber tenido excesivo éxito.

Desde los inicios de este paradigma de gestión, en la literatura especializada sobre dirección y gestión de empresas ha habido un amplio debate sobre la utilidad real de estos modelos, sistemas y estándares (véase, por ejemplo, Wilkinson, Godfrey y Marchington, 1997). De idéntica forma, en el ámbito sociosanitario se analizó -aunque quizá no con la atención y la profundidad debida- qué aportaban estos modelos al sector y cómo se integraban con otros modelos específicos de mejora y con las buenas prácticas profesionales relacionadas con la mejora de la calidad asistencial (Heras, Cilleruelo e Iradi, 2008). Tal como señalaba Heras (2008a), en el ámbito de los servicios sociales han existido planteamientos críticos que destacaban las debilidades de estos modelos 'generalistas' de gestión de la calidad, debilidades tales como el incremento de trabajo administrativo y la burocracia interna que se genera en un sector con una intensidad de trabajo ya de por sí muy grande, o el insuficiente potencial de estos modelos para la mejora de la calidad del servicio o la calidad asistencial que se ofrece al usuario en las organizaciones del sector. Con todo, este debate crítico no tuvo excesiva repercusión, al menos en la fase de fuerte crecimiento del paradigma de la gestión de la calidad. Con el estallido de la intensa crisis económica acaecido en 2008 , la cual ha propiciado tantos recortes en el gasto de las administraciones públicas, la extraordinaria difusión de modelos y sistemas se ha frenado en cierta medida, pero sin una reflexión de fondo sobre su orientación y sentido.

En la Comunidad Autónoma del País Vasco (CAPV), el paradigma de la gestión de la calidad se afianzó con una intensidad extraordinaria en los distintos sectores de actividad, tanto en los industriales como en los de servicios (Heras, Cilleruelo e Iradi, 2008). Así, se trata de una de las comunidades autónomas del Estado con mayor intensidad de certificación de la norma ISO 9001. La intensa labor promotora y tractora llevada a cabo por los distintos estamentos de las administraciones públicas vascas resultó un factor muy relevante para tal fin. Este hecho fue especialmente notorio en el sector sociosanitario, en general, y en el subsector de los servicios residenciales para personas mayores, en concreto. En la actualidad, se puede afirmar que en el sector de las residencias para personas mayores se está desarrollando de manera bastante intensiva y estructurada la estrategia de gestión global de este tipo de organizaciones. Sin embargo, existe una gran disparidad en los modelos de atención, los propios sistemas de recogida de información y las diferentes herramientas de medición de resultados. Parece que son muchos los centros donde la implantación de sistemas de gestión de la calidad bajo el estándar ISO 9001 no ha tenido la suficiente adecuación a su estructura. En consecuencia, algunos resultados obtenidos no parecen mostrar indicios de mejora de las organizaciones receptoras, sino una tendencia a cierto abandono, bien de la interiorización de algunos de los elementos básicos de estos modelos y referentes, bien del propio proceso de certificación o evaluación externa. Con todo, no hay, hasta la fecha, estudios que hayan tenido como fin analizar estos aspectos.

Pero no es algo que suceda únicamente en el sector que nos ocupa. Hasta fechas recientes, en la literatura teórica y empírica general no se ha prestado demasiada atención al fenómeno de la interiorización de la gestión de la calidad en sus diversas vertientes, tanto en lo que respecta a las distintas aproximaciones generales (aseguramiento, gestión de la calidad total, excelencia) como en lo que atañe a los principales modelos (como el estándar ISO 9001 y el modelo de autoevaluación EFQM). Salvo excepciones (véase, por ejemplo, Heras y Boiral, 2013), los estudios sobre la adopción de estos modelos no han analizado en profundidad el papel activo de los distintos agentes que conforman las organizaciones a la hora de adaptar y reformular en el día a día los principios e ideas que subyacen en los modelos y estándares de gestión conforme éstos son adoptados en entornos institucionales y culturales diferentes. Así las cosas, este trabajo tiene como objetivo analizar la adopción del estándar ISO 9001 en el ámbito de los servicios residenciales para personas mayores de la CAPV, y estudiar también cuáles han sido las causas y consecuencias del abandono de la certificación externa de estos sistemas de gestión de la calidad.

\section{Metodología y trabajo de campo realizado}

El presente estudio ha sido elaborado de forma conjunta y coordinada por un equipo investigador del Departamento de Organización de Empresas de la Universidad del País Vasco (UPV-EHU) y de la Univer- 
sitat de Girona. De cara a responder a las cuestiones planteadas, y tomando en cuenta los antecedentes sintetizados en el apartado anterior del artículo, se ha realizado un extenso trabajo de campo específico, en el que se han recabado los datos necesarios para alcanzar los resultados que aquí se presentan.

El trabajo de campo se desarrolló entre junio y noviembre de 2013, y tuvo tres componentes. Por un lado, se analizaron detenidamente las fuentes secundarias de información a través de diferentes recursos electrónicos, tanto buscadores especializados como generalistas. A su vez, se examinaron todo tipo de publicaciones y artículos científicos relativos a los modelos de gestión de la calidad en el sector sociosanitario y su implantación en él. En este orden de cosas, se realizó un trabajo recopilatorio de las principales publicaciones y manuales relacionados con la metodología de gestión por procesos y los modelos de atención residencial. Por último, se llevó a cabo una encuesta telefónica a 262 centros gerontológicos de la CAPV. Este estudio cuantitativo fue complementado, como se explicará a continuación, con un estudio de casos cualitativo.

La tasa de respuestas al cuestionario ascendió al $100 \%$ de los centros gerontológicos de la CAPV, ya que se realizaron diversas rondas de consulta, hasta que la totalidad de los centros colaboraron en el estudio. Vaya desde aquí, una vez más, nuestro agradecimiento más sincero a todas las personas que colaboraron en el estudio. En algunos casos, las llamadas fueron atendidas por el personal de secretaría y recepción de los centro, si bien en otros muchos las llamadas fueron derivadas a responsables de los correspondientes departamentos de calidad; en estos casos, las entrevistas fueron más extensas, de forma que se recogió una información más detallada y cualitativa. En ocasiones, las llamadas fueron atendidas por los mismos directivos de los centros.

Con el objeto de triangular la información obtenida, a lo largo del trabajo de campo se realizó un estudio cualitativo formado por once casos de organizaciones del sector que habían decidido abandonar la norma ISO 9001. La información necesaria se recabó mediante entrevistas semiestructuradas en profundidad a directivos, personal de atención directa y familiares de las residencias. Se utilizó un guión semiestructurado (Cuadro 1), pero suficientemente abierto, de tal forma que fuera coherente con el método inductivo de análisis de la información elegido y que, por consiguiente, no distorsionara la obtención de evidencias. En este sentido, el objetivo de las entrevistas y las consultas consistió en obtener narraciones de los interlocutores, más que respuestas concretas, tal y como plantea Yin (2003). A la hora de seleccionar las organizaciones objeto de estudio, el principal criterio fue su adecuación al propósito de la investigación; así, se eligieron aquellas residencias para personas mayores que, conforme a la información obtenida en las llamadas telefónicas previas, habían dejado de certificarse con la norma ISO 9001, motivadas por factores de diversa índole. Tal y como señala Boiral (2003), con el objetivo de evitar seleccionar organizaciones con insuficiente experiencia sobre la norma, todas las residencias que se eligieron tenían, al menos, cinco años de experiencia en la utilización de la ISO 9001 (la totalidad de residencias que habían dejado de certificarse con la norma cumplían este requisito).

Por otra parte, interesa señalar que gran parte de las entrevistas con el personal de atención directa y familiares se llevaron a cabo fuera de las residencias, tal y como se recomienda en la literatura especializada (por ejemplo, Boiral, 2003). Con el objetivo de no distorsionar la investigación inductiva, se evitó utilizar un discurso demasiado 'académico' o 'especializado' en las entrevistas. Las primeras entrevistas introductorias con el personal directivo de las residencias consultadas tuvieron una duración media de treinta minutos. Esta entrevista se repitió al cierre de la investigación, con una duración media muy similar a la anterior. Las entrevistas personales con el personal de atención directa, así como las realizadas

Cuadro 1. Resumen del guión de las entrevistas semiestructuradas

\section{Entrevistas a directivos}

¿Cuándo dejó de certificarse la residencia con la norma ISO 9001?

¿Por qué lo dejó?

¿Cuál fue el coste aproximado total de la implantación y su certificación?

¿Contaron con el apoyo de alguna empresa consultora?

¿Recibieron alguna ayuda o subvención para la implantación de la norma?

Aunque actualmente no cuenten con la certificación, ¿se ha interiorizado algún aspecto de la norma en el día a día del centro?

¿Trabajan actualmente con alguna de las siguientes herramientas?: manual de funcionamiento y calidad, carta de servicios, gestión del conocimiento, evaluación de la satisfacción, recogida de quejas y sugerencias, etc.

Entrevistas al personal de atención directa

¿En qué fase de implantación se encontraba la norma cuando usted se incorporó al centro?

¿Recibió alguna formación específica en relación al funcionamiento de la norma?

¿Supuso una carga de trabajo adicional excesiva la implantación de la norma?

¿Sigue trabajando actualmente con algún aspecto de la norma?

Entrevistas a los familiares

¿Cómo le comunicaron que el centro disponía de la certificación?

¿En qué aspectos mejoró la residencia?

¿Cree usted que el centro se diferenciaba de la competencia por tener dicha certificación?

Desde que el centro ha dejado de certificarse, ¿ha notado algún cambio?

Fuente: Elaboración propia. 
con los familiares, tuvieron una duración media de quince minutos. En total, se realizaron doce entrevistas semiestructuradas con directivos generales, trece con personal de atención directa y tres con familiares de los residentes.

\section{La implantación de la norma ISO 9001 en las residencias para personas mayores de la CAPV}

La norma ISO 9001 es un estándar internacional elaborado por la Organización Internacional para la Estandarización (ISO) que especifica los requisitos para un sistema de gestión de la calidad. No se trata de una norma que haga referencia al cumplimiento de un objetivo o a la consecución de un resultado, sino que implica la necesidad de documentar e implantar una metodología sistemática para la gestión en las organizaciones. La ISO 9001 tiene como finalidad la satisfacción de los clientes a través de una aplicación eficaz del sistema, incluidos los procesos para la mejora continua del sistema y el aseguramiento de la conformidad con todos los requisitos del cliente, así como de la reglamentación aplicable. Es, por tanto, un estándar que tiene una clara orientación a su aplicación en organizaciones que se dedican a la producción de bienes intermedios, donde existen unos requisitos técnicos muy claros que se suelen recoger, por ejemplo, en los pliegos de condiciones de los contratos. Con todo, hay que tener en cuenta que se trata de una norma genérica, que se puede aplicar en cualquier actividad o sector.
Esta norma no aporta ningún requisito específico para el sector residencial, pero incide en la necesidad de que las empresas realicen planes de mejora, identifiquen y monitoricen sus procesos a través de indicadores, describan los protocolos o procedimientos clave de su actividad, gestionen los recursos humanos y materiales, revisen periódicamente la eficacia del sistema en términos de mejora y analicen exhaustivamente la satisfacción de los usuarios principales del servicio que ofrecen. Establece, por tanto, un modelo de mejora continua general que puede ser de utilidad, por su carácter integral; tanto para la gestión como para la atención de las organizaciones residenciales.

En el Gráfico 1, se presenta el número de centros gerontológicos certificados en la CAPV de acuerdo con la norma ISO 9001. Según los datos obtenidos desde el Directorio de Entidades y Centros de Servicios Sociales ${ }^{2}$, en 2013 había un total de 262 centros en toda la comunidad autónoma, de los cuales aproximandamente el $56 \%$ se encontraban en Bizkaia; el $18 \%$, en Araba; y el $26 \%$, en Gipuzkoa. Por otra parte, en cuanto al número de centros certificados con esa norma se refiere, cabe destacar que el $47,33 \%$ de los centros analizados de la CAPV disponen actualmente del certificado ISO 9001. Así mismo, resulta reseñable que en el territorio histórico de Gipuzkoa, el 63,24\% de los centros ya se encuentre certificado, frente a un porcentaje de implantación algo inferior de Bizkaia, en concreto del 48,65\%. Llama también la atención igualmente el bajo grado de implantación que se ha dado hasta la fecha en Araba, donde llega muy tímidamente al $20 \%$ de los centros.

Gráfico 1. Centros gerontológicos certificados con ISO 9001. CAPV, 2013 (\%)

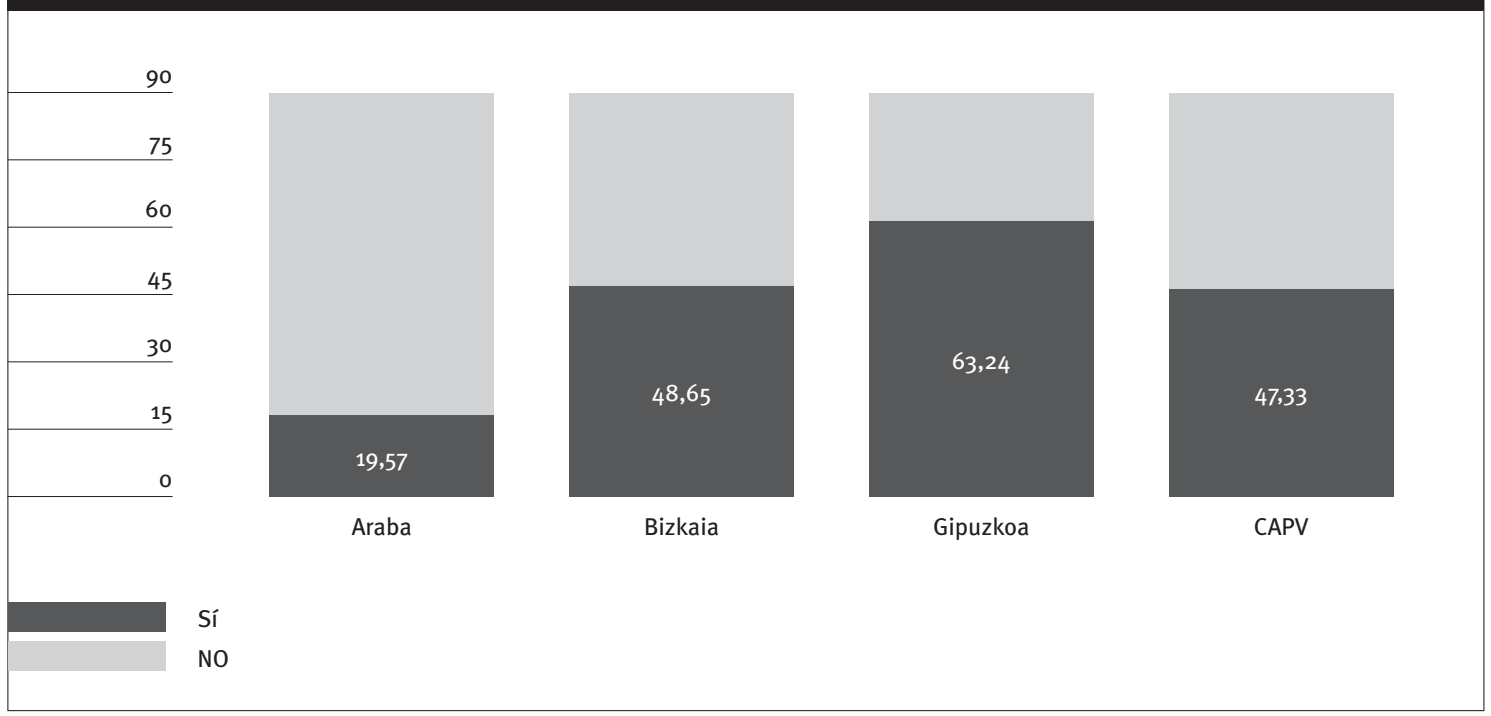

Fuente: Elaboración propia.

${ }^{2}$ El Directorio de Entidades y Centros de Servicios Sociales tiene como objetivo centralizar y ofrecer a toda persona interesada información relativa a entidades y centros que desarrollen su actividad en el ámbito de los servicios sociales en la CAPV. Se encuentra alojado en la dirección 〈http://www.direktorioa.net〉 y es de libre acceso. 
En el Gráfico 2, se muestra el número anual de primeras certificaciones obtenidas durante el periodo comprendido entre 1999 y 2013. Si bien es cierto que en estos últimos catorce años se observa que la mayor tasa de certificación tuvo lugar durante 2008 y 2009 , coincidiendo con la reciente puesta en marcha de la nueva versión de la norma ISO 9001:2008, es importante señalar que el número de primeras certificaciones obtenidas hasta ese momento supone un porcentaje acumulativo del 54,84\% respecto al total del periodo. Además, el incremento de esos dos años evidencia todavía más la bajada gradual que se ha identificado en el periodo comprendido entre 2008 y 2013.

Es evidente que las causas de esta reducción han podido ser diversas, tal y como se analizará seguida- mente. Con todo, en una primera aproximación señalaremos que la fuerte crisis económica que vivimos ha podido afectar a los centros residenciales, que en la actualidad cuentan, en términos generales, con unas menores tasas de ocupación y, por tanto, con menores recursos disponibles. La propia saturación del mercado ha posibilitado quizá también que los certificados ISO 9001 emitidos en los últimos años hayan disminuido porcentualmente.

Entre los principales motivos por los que las residencias optaron por implantar la citada norma (Gráfico 3), destacan la búsqueda de nuevas oportunidades de mercado y las exigencias impuestas por la administración pública. Dentro de las iniciativas institucionales, sobresale la del Departamento para la Política Social

Gráfico 2. Evolución del número de centros gerontológicos que obtienen su primera certificación ISO 9001, por año. CAPV, 1999-2013

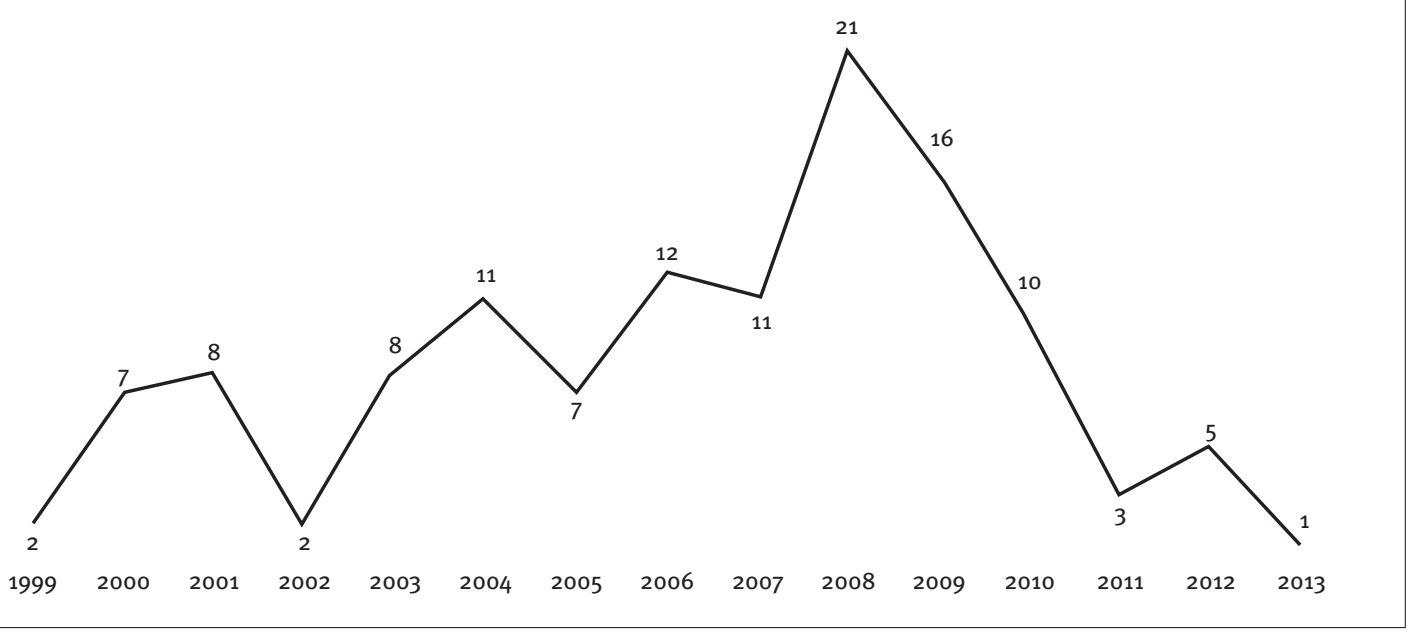

Fuente: Elaboración propia.

Gráfico 3. Motivación principal para implantar la norma ISO 9001 en los centros gerontológicos. CAPV, 2013 (\%)

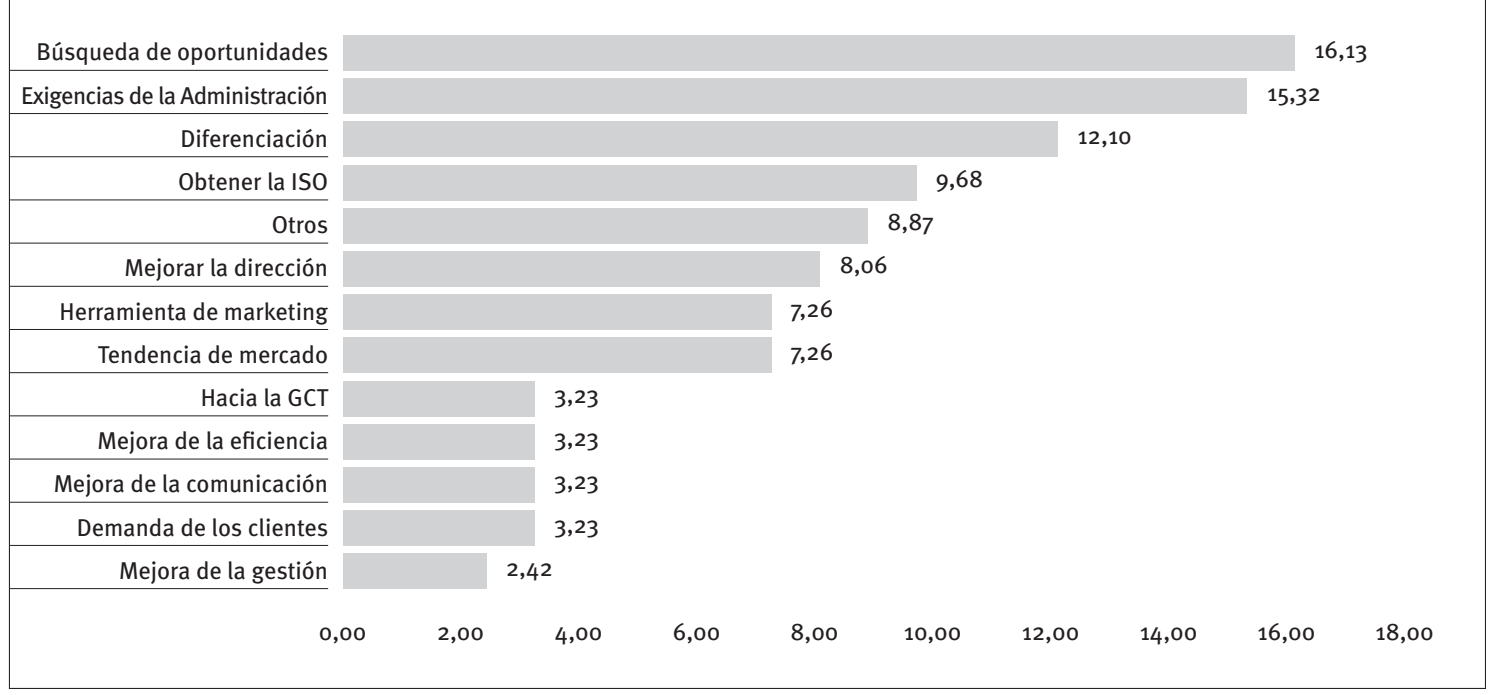

GCT: gestión de la calidad total.

Fuente: Elaboración propia. 
de la Diputación Foral de Gipuzkoa, que desarrolló en 2006 el catálogo de servicios residenciales que se debían implantar en todas las residencias de la Diputación, tanto públicas como privadas concertadas. El catálogo perseguía también asegurar la calidad asistencial y la homogeneización de esa calidad en todos los centros residenciales para personas mayores. Sin lugar a duda, esta y otras iniciativas constituyen un marco de trabajo que lleva a los centros a incorporar buenas prácticas y protocolos de atención, a gestionar sus resultados, e introducir el concepto de la gestión por procesos en las organizaciones.

Tal y como señalan Fontanals et al. (2007), en todo este proceso las administraciones públicas están jugando un papel muy importante. Sin embargo, parte de la dispersión y la diversidad se debe a las diferencias en los criterios estructurales y requisitos funcionales para la definición de los servicios asistenciales. Aunque se trate de una línea prioritaria, debería ir acompañada de otras medidas estructurales, como la mejora de la financiación, la renovación del sistema de subvenciones, la dotación de recursos profesionales y la mejora en la transparencia (Garau, 2005).

Un porcentaje muy similar de residencias (12,10\%) responden que la diferenciación ante la competencia fue una de las motivaciones más importantes para empezar a trabajar con el modelo. Paralelamente, un porcentaje cercano al $10 \%$ indica que la principal razón por la que optaron por la norma fue la propia certificación que se obtiene como fruto de adoptar el estándar. Se trata, en definitiva, de implantar un sistema de gestión de la calidad basado en el estándar internacional con el único fin de obtener el título organizacional, una actitud muy propia del llamado credencialismo organizativo descrito por Heras y Boiral (2013). Este aspecto podría estar estrechamente relacionado con otro tipo de motivaciones que se analizan con más detalle en el Gráfico 3: la demanda de los clientes o la consideración como una herramienta importante de marketing parecen tener un peso significativo a la hora de definir esta necesidad explícita por certificar los centros. De hecho, durante los últimos años se ha extendido el uso a todas luces inadecuado de las certificaciones, pues muchas organizaciones, aunque tan sólo certifican unos pocos de los procesos existentes $-y$ no, desde luego, los que más afectan a la calidad asistencial que ofrecen-, utilizan la certificación obtenida de una forma que podría asimilarse a la publicidad engañosa.

\section{Residencias que dejan de certificarse conforme a la norma ISO 9001}

Como se ha indicado anteriormente en la metodología del estudio, como punto de partida del trabajo de campo se realizó una primera aproximación a residencias que podrían estar certificadas, tomando como referencia para ello datos facilitados por los distintos organismos certificadores, tales como Aenor. Así, en la primera toma de contacto telefónica con aquellos centros que sí disponían de la corres- pondiente certificación, y al preguntar sobre ella, se recogieron en muchas ocasiones por parte del personal de las organizaciones consultadas - personal tanto de atención directa como indirectarespuestas que evidencian el carácter superficial de la implantación de la norma ISO 9001. Seguidamente se muestran algunos de los comentarios más alarmantes que evidencian un grado de interiorización muy reducido de la ISO 9001 en las organizaciones consultadas:

Yo no te lo podría decir, porque soy una auxiliar (auxiliar de enfermería de una residencia certificada desde 2004, 62 plazas).

No entiendo muy bien esas cosas. Mejor si llamas otro día y preguntas por la responsable de calidad (Servicio de Atención al Cliente de una residencia certificada desde 2001, 150 plazas).

Entiendo que estarás hablando sobre esas inspecciones que nos suelen realizar frecuentemente. Yo diría que sí la tenemos (personal del Servicio de Admisión de una residencia certificada desde 2009, 74 plazas).

No me aclaro con tantos papeles. Mejor si llamas otro día (auxiliar administrativo de secretaría de una residencia certificada desde 2003, 68 plazas).

Se trata tan sólo de una muestra, puesto que fueron muchas las respuestas obtenidas en esta misma línea. Entre las llamadas realizadas a todos los centros de la CAPV (262), se identificaron 11 residencias para personas mayores que dejaron de certificarse con la norma ISO 9001. Según el estudio, 72 centros gerontológicos de Bizkaia se encuentran actualmente certificados. En este sentido, la tasa de abandono de la certificación de este territorio histórico es muy superior a las identificadas en Gipuzkoa y Araba. Concretamente, son 6 las residencias que dejaron de certificarse en Bizkaia, frente a 3 de Gipuzkoa y 2 de Araba, respectivamente. Por otra parte, aunque el porcentaje de implantación en Gipuzkoa (63,24\%) sea muy superior a la de Bizkaia $(48,65 \%)$, tal y como se ha señalado anteriormente, son sólo 2 los centros guipuzcoanos que han dejado de certificarse.

En el Cuadro 2 se sintetizan algunos datos descriptivos de las organizaciones analizadas en el estudio de casos, como información relevante sobre la dimensión de la organización, así como sobre la motivación inicial, de acuerdo a la opinión manifestada por el director o coordinador del área de calidad. Se especifica también si la organización analizada utiliza en el día a día, según las personas entrevistadas, algunas de las herramientas o técnicas que mayor aceptación han tenido en el sector residencial. En concreto, se analiza si se utilizan herramientas tales como el cuadro de mando integral, el diagrama de flujos, el diagrama de causa-efecto, los cuestionarios de satisfacción, los equipos o grupos de mejora, las ${ }_{5} \mathrm{~S} 0$ las cartas de servicios. 


\begin{tabular}{|c|c|c|c|c|c|c|c|c|c|c|c|c|}
\hline \multicolumn{13}{|c|}{ Cuadro 2. Perfil de los centros visitados } \\
\hline \multirow[b]{2}{*}{$\begin{array}{l}\text { Territorio } \\
\text { histórico }\end{array}$} & \multirow[b]{2}{*}{ ID } & \multirow[b]{2}{*}{ Plazas } & \multirow[b]{2}{*}{$\begin{array}{l}\text { Primera } \\
\text { certificación } \\
\text { (año) }\end{array}$} & \multirow[b]{2}{*}{ Motivación inicial } & \multicolumn{8}{|c|}{ Herramientas interiorizadas } \\
\hline & & & & & 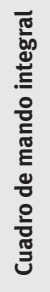 & $\begin{array}{l}\frac{n}{3} \\
\frac{0}{3} \\
\frac{0}{0} \\
\frac{\pi}{0} \\
\frac{\pi}{5} \\
\frac{\pi}{00} \\
\frac{\pi}{0}\end{array}$ & 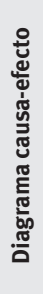 & 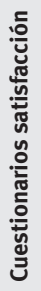 & 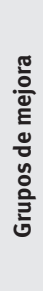 & 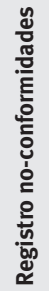 & n & 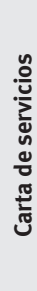 \\
\hline \multirow{2}{*}{ Álava } & $\mathrm{A} 1$ & 50 & 2001 & Diferenciación de los competidores & $\mathrm{x}$ & $x$ & $\mathrm{x}$ & $x$ & $x$ & & & \\
\hline & $\mathrm{A} 2$ & 24 & 2003 & $\begin{array}{l}\text { Búsqueda nuevas oportunidades } \\
\text { de mejora }\end{array}$ & & $x$ & & $x$ & & $\mathrm{x}$ & & \\
\hline \multirow{6}{*}{ Bizkaia } & $\mathrm{B} 1$ & 27 & 2002 & \multirow{2}{*}{ Conseguir la certificación } & & $x$ & & $\mathrm{x}$ & & & & \\
\hline & B2 & 17 & 2006 & & & $x$ & & $x$ & & & & \\
\hline & B3 & 22 & 2000 & $\begin{array}{l}\text { Satisfacer exigencias } \\
\text { de la Administración }\end{array}$ & & $\mathrm{x}$ & & $x$ & $x$ & $x$ & & \\
\hline & B4 & 25 & 2005 & Herramienta de marketing & & $x$ & & $\mathrm{x}$ & & & & \\
\hline & $\mathrm{B}_{5}$ & 66 & 1999 & Mejorar la gestión & $x$ & & & $\mathrm{x}$ & $x$ & $\mathrm{x}$ & $\mathrm{x}$ & $\mathrm{x}$ \\
\hline & B6 & 20 & 2001 & $\begin{array}{l}\text { Búsqueda de nuevas oportunidades } \\
\text { de mejora }\end{array}$ & & $x$ & & $x$ & & & & \\
\hline \multirow{3}{*}{ Gipuzkoa } & $\mathrm{G}_{1}$ & 32 & 2001 & Tendencia del mercado & & & & $\mathrm{x}$ & & & & \\
\hline & $\mathrm{G}_{2}$ & 14 & 2000 & $\begin{array}{l}\text { Herramienta } \\
\text { de marketing }\end{array}$ & & $\mathrm{x}$ & & $\mathrm{x}$ & & & & \\
\hline & $\mathrm{G}_{3}$ & 120 & 2003 & Mejorar la eficiencia & $\mathrm{x}$ & & & $x$ & $x$ & $x$ & $x$ & $x$ \\
\hline
\end{tabular}

ID: código de identificación.

Fuente: Elaboración propia.

A través del cuadro de mando integral (también conocido como Balanced Scorecard Dashboard), la organización puede monitorizar sus objetivos. Se trata de una herramienta que ayuda a las organizaciones a expresar las iniciativas necesarias para el cumplimiento de sus estrategias. El diagrama de flujos (también denominado diagrama de actividades) representa gráficamente los procesos de la organización, utilizando para ello símbolos con significados definidos que representan los pasos del proceso. Por otra parte, mediante el uso de cuestionarios de satisfacción las organizaciones recaban información sobre el grado de cumplimiento de las expectativas de sus clientes ante un servicio ofrecido o la valoración de un conjunto de circunstancias. A su vez, los grupos de mejora tienen como misión identificar, analizar y proponer soluciones a ineficiencias del propio trabajo 0 actividad. En cuanto al método de las ${ }_{5} \mathrm{~S}$ (así denominado por la primera letra del nombre que en japonés designa cada una de sus cinco etapas), es una técnica de gestión japonesa basada en estos cinco principios simples: clasificación, orden, limpieza, estandarización y mantener la disciplina. Por último, las cartas de servicios se han convertido en una de las herramientas más utilizadas, tanto por las administraciones públicas como por las empresas privadas, para comunicar a sus usuarios los servicios que prestan y los compromisos que asumen en su prestación, y constituyen una iniciativa fundamental para la modernización de la gestión de numerosas organizaciones; así, cada vez son más las organizaciones de servicios sociales que tienen establecidas sus cartas de servicios (Heras y Martiarena, 2007).

La mayoría de las personas con un cargo directivo entrevistadas reconocieron que gracias a la introducción de ideas básicas asociadas a la ISO 9001, como la mejora continua, se observaron cambios muy significativos en la gestión de la residencia, de forma que se fueron incluyendo acciones y medidas que hasta entonces no se llevaban a cabo, tales como la redacción de algunos procedimientos muy significativos de su funcionamiento para garantizar que todo el equipo de trabajo los conociera y actuara de forma más homogénea, evitando así la variabilidad y la improvisación en los procesos. En el discurso de los directivos entrevistados que valoraban los resultados iniciales de la adopción de la ISO 9001, también se constata cierta retórica empresarial, cierta retórica del éxito, ya evidenciada en otros estudios (Zbaracki, 
1998; Heras y Boiral, 2013). Entre los comentarios y las observaciones que se recogieron en este tono entre los directivos, destacan las siguientes:

Los requerimientos del mercado son cada vez más rígidos, y vimos que era necesario replantear algunos de los procesos, la forma de la empresa, la estructura y todo lo que intervenía en la prestación del servicio. Sabíamos hacer bien las cosas, pero reconocíamos que los procesos no eran eficientes y rigurosos (residencia G2).

Buscábamos una distinción ante las residencias del sector. Nos permitía [una] mayor capacidad de negociación con nuestro principal cliente (residencia $\mathrm{B}_{3}$ ).

El objetivo no debería haber sido conseguir la certificación; debíamos haber utilizado la norma para alcanzar la excelencia. Optamos por la certificación por lo que otorgaba, y no por lo que significaba. Además, nuestra estrategia comunicativa se centró en presentar al exterior la implantación de la ISO [9001] (residencia B1).

Se realizaba muy poco trabajo en grupo. Sabíamos que realizar ciertas gestiones eran importante, pero desconocíamos el motivo. Se nos controlaba en todo momento, pero no se nos ofrecía ninguna formación específica de cómo mejorar en nuestras labores (residencia B2).

Los equipos de trabajo multidisciplinares de parte de las residencias entrevistadas coincidían en que la implantación del estándar sirvió para ordenar las ideas respecto a lo que necesitaban mejorar. El modelo les ayudó en la búsqueda proactiva de nuevas potencialidades en la organización, nuevas posibilidades de cambio y mejora. Además, la adopción del estándar facilitó la introducción de otras innovaciones organizativas en las residencias como, por ejemplo, la gestión por procesos.

Sin embargo, un aspecto muy común en la mayoría de las personas entrevistadas en los centros visitados es la sensación de inutilidad por no comprender lo que se pretendía hacer con algunos elementos que componían el modelo. Aunque esta perspectiva estaba más presente entre los trabajadores de atención directa, varios directivos admitieron que atribuían al sistema de gestión de la calidad más exigencias de las que realmente planteaba. Además, coincidían en que muchas de éstas venían de la forma en que la empresa consultora que apoyó a los centros interpretaba el propio sistema de calidad de la residencia. En la misma línea, la mayor parte de los empleados de atención directa señalaron que frecuentemente se resignaban a mantener algunos elementos del modelo ISO 9001 que entendían que eran totalmente innecesarios y burocráticos, de manera que hipotecaban el proceso de cambio de la residencia hacia la calidad, ya que asociaban inicialmente la calidad a algo que no aportaba nada más que imagen, en su caso. En este sentido, se recogen a continuación algunas reflexiones realizadas por parte del personal de atención directa entrevistado en torno al proceso de implantación e interiorización del sistema de gestión de la calidad:

Se inició el proceso de implementación sin antes realizar el debido proceso de sensibilización (residencia A2).

Cuando la dirección nos comunicó que la residencia había obtenido la certificación, nos sentimos muy orgullosos de todo el trabajo realizado previamente; fue un reconocimiento a nuestra labor (residencia $B_{5}$ ).

Somos una empresa de reciente constitución, y la organización del equipo no es todavía muy madura. Además no teníamos una orientación hacia el trabajo en equipo. La dirección no nos informó [de] en qué consistía la ISO, y tampoco [de] qué papel tendríamos en la implantación de la misma. La terminología de los documentos era muy técnica. El excesivo tiempo que nos llevaba rellenar las fichas de seguimiento de cada caso nos hizo dejar de lado otros programas de psicoestimulación que habíamos puesto en marcha con anterioridad y que funcionaban con mucho éxito (residencia B4).

Por otra parte, se realizaron entrevistas individuales con familiares de los propios residentes. Si bien en la mayor parte de los casos los entrevistados no estaban al corriente de lo que suponía la implantación y certificación de la ISO 9001 en la organización donde se atendía a sus parientes, sí que se obtuvieron algunos comentarios de interés por parte de familiares que, en mayor o menor medida, estaban al corriente de lo que supone la adopción de la ISO 9001. He aquí algunas respuestas que se recogieron en relación a las preguntas anteriormente presentadas:

El centro, tras la certificación, quedó igual o peor, pero con un certificado (residencia B2).

Me dijeron que, debido a la implantación del estándar, mis exigencias no tendrían una solución inmediata, porque la ISO [9001] les suponía un procedimiento muy largo (residencia $\mathrm{B} 2$ ).

Las soluciones que planteaba el centro ante los problemas de nuestros familiares carecían de cualquier tipo de enfoque a medio/largo plazo; no se analizaba qué incidencia podría tener una actuación concreta en el bienestar de la persona mayor. Notábamos situaciones de tensión [se entiende que relacionadas con la adopción de la ISO 9001], porque unos se culpaban a otros por los errores (residencia B4).

Los problemas de satisfacción del residente siguen repitiéndose, y en muchos casos son hasta peores (residencia $\mathrm{G}_{2}$ ).

Muchas de las personas entrevistadas, la mayoría de atención directa, subrayan que, para muchos familia- 
res, se ha evidenciado que su centro gerontológico certificado con la ISO 9001 no daba mejor servicio que otros que no tenían dicha certificación, lo cual demuestra, según estas personas, que los centros sin certificación podrían tener un sistema de gestión de la calidad tan eficaz como la suya, incluso desconociendo el contenido y los requisitos de norma ISO 9001.

En los casos analizados, se ha puesto de manifiesto que las organizaciones residenciales optan por dejar de certificar la ISO 9001 fundamentalmente debido a dos motivos: por un lado, por razones de índole económica y, por otro, por el reconocimiento externo concreto que la norma ISO 9001 tiene para los potenciales usuarios de las residencias y, en mayor medida, para las administraciones públicas. En lo que respecta al primer factor, se ha de reseñar que muchas de las residencias entrevistadas resaltaron el alto coste que les suponía la certificación de la norma y su mantenimiento. Cabe señalar que, sin tener en cuenta aspectos relacionados con las certificaciones o las evaluaciones externas, el coste de la implantación de la ISO 9001 viene determinado por el tiempo que la organización pueda dedicarle y por los gastos directos que pueda generar. Así mismo, desde la perspectiva de las certificaciones, para la obtención de una certificación ISO 9001 la residencia tiene que abonar los costes de la empresa certificadora y, aunque no sea estrictamente necesario, de una empresa consultora que le ayude a ponerla en marcha.

En lo que respecta al reconocimiento externo de la norma ISO 9001, cabe señalar que un número significativo de las personas entrevistadas también coincidían en que la saturación del mercado ha facilitado la pérdida de reconocimiento de la ISO 9001, al haberse popularizado en exceso y haberse producido una clara erosión en su imagen respecto a la que tenía hace diez años en el sector. Muchas organizaciones del sector sociosanitario pioneras en la certificación de la ISO 9001 han adoptado modelos de calidad total como el modelo de autoevaluación EFQM, que, en líneas generales, se ve como un modelo más exigente, tanto en la literatura académica como profesional (Casadesús, Heras y Merino, 2005).

Por otra parte, cabría hacer referencia al efecto que los cambios en las distintas iniciativas de las administraciones públicas han podido tener en la renuncia a la renovación de las certificaciones ISO 9001 en el sector objeto de atención. En este sentido, se ha de tener en cuenta que dicho impulso de las administraciones públicas se manifiesta al menos en dos direcciones: por una parte, como demandante de servicios concertados, dichas administraciones pueden -como ocurre en muchos otros sectores de actividadreconocer o valorar, de forma más o menos directa o velada, a las organizaciones residenciales que cuentan con este tipo de certificaciones; y por otra, pueden promover su adopción a través de diversos mecanismos, como las ayudas y las subvenciones directas a la implantación y certificación de este tipo de estándares, los programas específicos para el impulso de dichos modelos de gestión, la publicación y promoción de modelos específicos adaptados al sector, o la promoción de adopciones en grupos de organizaciones.

En este último caso, parece evidenciarse que en los últimos años se ha producido una reducción en el impulso dado por parte de las administraciones públicas en el sector, de acuerdo a lo manifestado por algunas de las organizaciones consultadas, cuestión esta que también se evidenciaba para el conjunto de la CAPV en el informe dirigido por Heras (2008b) para Orkestra-Instituto Vasco de Competitividad. En concreto, se nos señaló que desde hace unos años no se concedían subvenciones específicas a la certificación ISO 9001 si no se contemplaba también la adopción de un sistema de gestión ambiental de acuerdo a la norma ISO 14001, un factor que puede repercutir en el abandono de este tipo de certificaciones de cara al futuro.

A la hora de justificar los motivos por los que las organizaciones residenciales decidieron no renovar los certificados ISO 9001, los directivos entrevistados manifestaron razones muy diversas, aunque gran parte de ellas se centraban en críticas más o menos explícitas a la forma en la que se implantó el modelo de gestión de la calidad. He aquí algunos ejemplos al respecto:

No hubo compromiso suficiente por parte de todos los responsables de los equipos multidisciplinares del centro. La residencia carecía de un mínimo de procesos ya definidos, no había tenido ninguna experiencia previa similar. El estándar no era totalmente aplicable a la realidad de la empresa, ya que no considera la singularidad del sector ni de las propias personas mayores (residencia $\mathrm{G}_{3}$ ).

No hemos sabido asimilar la filosofía del estándar (residencia $\mathrm{B}_{4}$ ).

Existía una departamentalización excesiva. Las áreas funcionales tenían muy poca orientación hacia el trabajo integral (residencia G3).

El efecto de la ISO 9001 fue entendido por los trabajadores como otra tendencia organizacional más que pasa de moda (residencia G1).

La ISO dejó de ser un valor agregado al sistema de mejora de la calidad y se convirtió en un serio problema que afectaba al ambiente de la organización (residencia A1).

Con todo, sí que se constató que, a pesar de no renovar la certificación ISO 9001 parte de las organizaciones residenciales analizadas, éstas continuaban manteniendo algunos de los elementos propios de un sistema de gestión de la calidad conforme al referente internacional. Así mismo, se han añadido otras aportaciones sobre el funcionamiento actual de la gestión (propiamente dicha), tras el abandono de la certificación de la norma. 
Aunque ya no sigamos con la ISO, ahora mi participación en los grupos de trabajo interdisciplinar es mayor. Tengo más claro cuál es mi responsabilidad, y en base a ella ejerzo mis funciones. [...] Cuando participo en las reuniones semanales de control de los usuarios, me siento motivada para exponer mis inquietudes y propuestas de mejora para el futuro de la residencia. Se ha dejado atrás la tendencia de la improvisación. Podemos plantear nuestras dudas e ideas con total tranquilidad. Muchas de nuestras propuestas son consideradas y puestas en marcha en la atención diaria (residencia $G_{3}$ ).

Ahora, una vez que hemos dejado de certificarnos, en cuanto a la orientación de los resultados, seguimos trabajando con un sistema que refleja los logros, y en este sentido, las mediciones son semanales. Se ha apostado por una cultura proactiva: los trabajadores aportan ideas novedosas que, a su vez, se interiorizan en la propia gestión de la residencia para una mejora continua de la prestación del servicio. [...] No todos los procesos fueron estandarizados, pero por lo menos se identificaron aquellos que más valor generaban al residente. Por otra parte, ahora conocemos más a nuestros clientes. Estamos más abiertos al cambio. Seguimos ampliando la implantación de las $5 \mathrm{~S}$ en diferentes almacenes del resto de la residencia. Estamos procurando estandarizar toda la documentación nueva que se está generando, después de la puesta en marcha del centro de día (residencia B5).

Tenemos claro que se necesita alinear la gestión de la calidad con la estrategia de negocio del centro. Ahora entendemos el sistema de gestión de la calidad como fuente que genera fortalezas, permitiendo la disminución de los costos o incremento de los ingresos, dos variables muy importantes de la productividad de nuestra organización (residencia $\mathrm{A} 2$ ).

Nos ha quedado claro que dos principios clave para potenciar la satisfacción y el desempeño de las personas son el liderazgo y el compromiso de la dirección. Además, hemos aprendido que la gestión de la calidad debe suponer para la empresa una orientación hacia los procesos y no hacia las funciones. Aunque ya no dispongamos de ninguna certificación, intentamos identificar todo tipo de mecanismos de mejora continua del sistema (residencia $\mathrm{A} 1$ ).

A partir de los datos obtenidos sobre el grado de interiorización de las diferentes herramientas y aspectos de la ISO 9001, se ha procedido a clasificar la interiorización de acuerdo a los tres niveles siguientes. El primero recogería aquellas organizaciones que, tras haber dejado de certificarse, no siguen trabajando de acuerdo a los principios de la norma, y en consecuencia no muestran indicios de hacer uso de sus propias herramientas. La mayoría de estas organizaciones no están convencidas de que la aplicación del modelo les sea útil, más bien la ven como una fuente de trabajo extra. Además, no todo el equipo piensa que la aplicación del sistema de gestión de la calidad haya propiciado las mejoras observadas, pues algunas personas creen que la mejora es lo que habitualmente sucede cuando el personal hace bien su trabajo. Podrían clasificarse dentro de este grupo las residencias $\mathrm{B}_{1}, \mathrm{~B} 2$, B4 y B6, con una dimensión de entre 15 y 25 plazas. Tal y como se recoge en el Cuadro 2, lo único que han interiorizado estos centros es el uso de cuestionarios de satisfacción y diagramas de flujos. Si bien se trata de herramientas con una gran utilidad para el propio sistema de gestión de la calidad, la información debería complementarse, por ejemplo, mediante grupos de mejora o el análisis a través de diagramas causa-efecto. Cabe destacar que la motivación inicial de estas residencias para la implantación de la ISO 9001 se limitó a la propia certificación de la norma y a su uso como herramienta de marketing.

El segundo grupo recogería aquellas organizaciones en las que, tras el abandono de la certificación de la norma, se han interiorizado varias de las herramientas analizadas. En estas organizaciones, los empleados coinciden en que la aplicación de un sistema de gestión de la calidad les ayuda a organizarse y a ordenar la gestión de los procesos. No obstante, también coinciden en que ello representa una mayor carga de trabajo, al tener que completar y usar un mayor número de documentos y registros de calidad. Así mismo, creen que el cumplimiento total de lo programado es muy difícil, debido a un sinfín de imprevistos. En este mismo orden de cosas, muchas de estas empresas piensan que es suficiente seguir paso a paso las indicaciones de la ISO 9001 para conseguir una mejora en la gestión. Sin embargo, un sistema de gestión de la calidad requiere un cambio en la forma de enfocar la gestión de las empresas y supone centrarse en las necesidades del usuario. Es más, de las entrevistas realizadas a los centros se concluye que el enfoque al cliente es uno de los principios que el personal percibe con el más bajo cumplimiento. Esto se debe a que el personal de estas organizaciones sigue las indicaciones de una norma que perciben que no se adapta a sus necesidades. En el fondo, se podría decir que sus sistemas de gestión de la calidad funcionan perfectamente en lo que se refiere a la teoría, pero en la práctica, tienen varias deficiencias: no todo el personal tiene las mismas metas finales, y esto se traduce en diferentes percepciones de calidad de cada una de estas personas. Así las cosas, las residencias $\mathrm{A}_{2}$ y $\mathrm{B}_{3}$ (con aproximadamente 25 plazas) podrían clasificarse dentro de esta categoría.

El tercer y último grupo de clasificación de centros recogería aquellas residencias que, tras el abandono de la certificación, siguen trabajando activamente con la mayoría de las herramientas puestas en marcha durante el proceso de implantación y funcionamiento de la norma. Cabe referirse, por ejemplo, a indicadores de gestión asimilables al cuadro de mando integral, los diagramas de flujos, los diagramas de causa-efecto, los cuestionarios de 
satisfacción y al registro de no conformidades. Sin embargo, debería incidirse en la importancia del uso de herramientas como las cartas de servicio y las $5 \mathrm{~S}$, fundamentales en el sistema de gestión de la calidad. En este sentido, las residencias $A_{1}, B_{5}$ y $G_{3}$, todas ellas con una dimensión superior a las 50 plazas, son las que han interiorizado de manera más intensiva los principios y herramientas de la ISO 9001.

\section{Conclusiones}

A lo largo del trabajo de campo se ha constatado que la norma ISO 9001, uno de los principales referentes internacionales para la gestión de la calidad, ha tenido una amplia difusión en las organizaciones de servicios residenciales para personas mayores de la CAPV. También ha detectado que, a pesar de la situación de crisis generalizada y los momentos de dificultad que está pasando el sector, la tasa de abandono de la certificación ISO 9001 es reducida. Tan sólo once centros gerontológicos de la CAPV han dejado de certificarse durante los últimos quince años. Asimismo, y a pesar de tener una presión externa similar para su adopción, incluso en las organizaciones residenciales que han decidido no renovar la certificación ISO 9001, su interiorización ha sido heterogénea.

La adopción de la norma ISO 9001 parece que ha resultado de cierta utilidad para las organizaciones del sector sociosanitario, en general, y para las del sector de prestación de servicios residenciales para personas mayores, en concreto. Asimismo, esta corriente ha ayudado a que las organizaciones reflexionen también en términos operativos, pues son muchas las que se han dotado de indicadores, programas y herramientas de mejora concretas. La difusión de este y otros modelos de calidad ha servido, además, para profesionalizar la gestión y equipararla a la de otros sectores de actividad. Ha servido, por decirlo de alguna forma, para que las organizaciones del sector utilicen el lenguaje y los modelos de gestión y dirección de organizaciones convencionales, lo que representa un factor motivador para muchas personas involucradas en la gestión, entre otros motivos porque, al utilizar sistemas, modelos y herramientas comunes a otros sectores de actividad, se han visto equiparadas u homologadas a organizaciones de otros sectores, como el industrial, que se han caracterizado siempre por una mayor legitimidad social y un reconocimiento de eficiencia superior.

Ahora bien, tal y como se ha constatado en el trabajo de campo sintetizado en este artículo, toda esta corriente de modelos, sistemas y herramientas para la mejora de la calidad también ha tenido sus claroscuros. En muchas organizaciones del sector el modelo de calidad no se ha interiorizado de forma adecuada, de manera que la adopción de un sistema para la gestión de la calidad se ha entendido como una actividad funcional específica de la empresa a cargo de unas personas determinadas. Dicho en otras palabras, se ha entendido a modo de 'una actividad extra', en lugar de como una función transversal dentro de la actividad de dirección y gestión de la organización. Así, la adopción de la ISO 9001 parece haberse convertido en muchos casos en un fin en sí mismo, más que en un medio para mejorar la gestión de la organización, y, quizá más importante aun, mejorar la calidad del servicio y la calidad asistencial que ofrecen las organizaciones residenciales a los usuarios.

Una vez más, se constata que algunos de los beneficios potenciales de los sistemas y modelos para la calidad están muy relacionados con la forma en la que se adoptan. Así, las personas de las distintas organizaciones que incorporan la norma ISO 9001 son actores activos que, en su labor, reconstruyen, reconfiguran y reinterpretan el modelo que trata de definir el estándar internacional, adaptándolo a la cultura de su organización y a su actividad. Así, en un sector de actividad como el de la prestación de servicios residenciales a personas mayores, la adhesión del personal de atención directa que produce el servicio clave a las personas usuarias en la adopción de estos modelos resulta vital. Por consiguiente, sin una interiorización adecuada, la norma ISO 9001 corre un serio peligro de convertirse en un mero título organizacional, un simple certificado que no aporta valor real a la organización que lo adopta. Es más, puede resultar incluso perjudicial, por el trabajo extra que puede suponer, el aumento de la intensidad de trabajo, la presión de las auditorías externas y por otros factores relacionados.

En el ámbito de los servicios residenciales para personas mayores parece evidente que la difusión de la norma ISO 9001 se ha debido, en gran medida, al fuerte empuje de las administraciones públicas, que pueden jugar un papel relevante a la hora de promocionar unos modelos de gestión determinados respecto a otros. Pasada ya una década desde que, junto con la activa colaboración de otros agentes promotores y prescriptores de estos estándares de gestión (tales como asociaciones, organismos certificadores o consultorías), las administraciones públicas decidieran promover la adopción de estas normas ISO 9001 como referente para la mejora de las organizaciones del sector quizá haya llegado el momento de que se realice una reflexión en profundidad sobre los efectos reales de esta política de promoción. Además esta cuestión resulta más importante, si cabe, en un entorno económico y empresarial caracterizado por una fuerte crisis económica, fruto de la cual se están implementando severas medidas de ajuste en la capacidad de inversión y gasto en el sector sociosanitario. 


\section{Referencias bibliográficas}

BOIRAL, O. (2003): "ISO 9000, outside the iron cage", Organization Science, vol. 14, no 6, págs. 720737.

CASADESÚS, M.; HERAS, I.; y MERINO, J. (2005): Calidad práctica, Madrid, Pearson Educación, Madrid.

FONTANALS et al. (2007): Calidad y dependencia. Grados de dependencia y necesidad de servicios, Madrid, Imserso.

GARAU, J. (2005): Guía para la gestión de calidad de los procesos de servicios sociales, Madrid, Intress; Madrid, Ministerio de Trabajo y Asuntos Sociales.

HERAS, I. (2008a): “Calidad sustantiva versus calidad superficial”, Gizarte.doc, no 26, pág. 7 [ [khttp:// www.siis.net/documentos/gizarte/Numero26. pdf\#page $=7>$ ].

- (dir.) (2008b): Gestión de la calidad y competitividad de las empresas de la CAPV, Bilbao, OrkestraInstituto Vasco de Competitividad; Bilbao, Universidad de Deusto.

HERAS, I.; y BOIRAL, O. (2013): "Symbolic adoption of ISO 9000 in small and medium-sized enterprises: The role of internal contingencies", International Small Business Journal [en prensa].

HERAS, I.; CILLERUELO, E.; e IRADI, J. (2008): “Quality management and quality of care in nursing homes", International Journal of Health Care Quality Assurance, vol. 21, n- 7, págs. 659-670.

HERAS I.; y MARTIARENA, A. (2007): “Zerbitzu-kartak eta zerbitzuen kalitatearen hobekuntza", Zerbitzuan, no 42, págs. 39-44 [rhttp://www. zerbitzuan.net/documentos/zerbitzuan/ Zerbitzu\%2okartak\%2oeta\%2ozerbitzuen\%20 hobekuntza.pdf〉].

WILKINSON, A.; GODFREY, G.; y MARCHINGTON, M. (1997): "Bouquets, brickbats and blinkers: Total quality management and employee involvement in practice", Organization Studies, vol. 18, nํㅜ 5, págs. 799-819.

YIN, R. K. (2003): Case Study Research: Design and Methods, Thousand Oaks, Sage Publications.

ZBARACKI, M. J. (1998): "The rhetoric and reality of total quality management", Administrative Science Quarterly, vol. 14, nํ․ 43, págs. 602-636. 